\title{
Sacral insufficiency stress fracture as etiology of positional autonomic dysreflexia: Case report
}

\author{
JP Beard, WH Wade ${ }^{1}$ and DB Barber

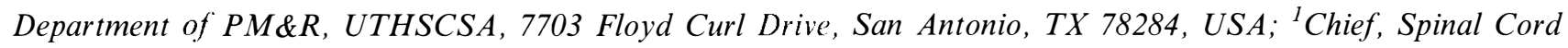 \\ Injury Service, Audie L Murphy Veterans Administration Hospital
}

\begin{abstract}
The medical literature is replete with case reports of the syndrome known as autonomic dysreflexia. Although the majority of cases are known to be induced by either bladder or bowel distention, there does exist a small number of cases in which the inciting stimulus is more obscure. In such cases, a comprehensive medical evaluation is necessary to ensure proper identification of the source of irritation and the appropriate medical management of the patient. We present a patient with a heretofore unreported suspected etiology of autonomic dysreflexia, axial loading of a sacral stress fracture.
\end{abstract}

Keywords: autonomic dysreflexia; spinal cord injury; tetraplegia; stress fracture

\section{Case report}

A 41 year old male with a traumatic C5 complete tetraplegia of 25 years duration presented to the spinal cord injury unit for his annual physical examination and evaluation of recurrent urinary tract infections (UTIs). The patient was known to have a neuropathic bladder secondary to his spinal cord injury (SCI) and had undergone three previous sphincterotomies, utilizing a condom catheter for bladder drainage. He also had a neuropathic bowel for which a digital stimulation and suppository bowel program was used every three days without difficulty.

The patient had no medical complaints on admission. His physical examination was consistent with a $\mathrm{C} 5$ complete tetraplegia. His laboratory results: (complete blood count (CBC), chemistry and prostatespecific antigen) were unremarkable. Following admission, a routine urodynamic study was performed which revealed a large-capacity, low pressure bladder. The urological recommendation was for placement on an intermittent catheterization program, however, the patient instead consented to the use of an indwelling Foley catheter.

On the day following the urodynamic study, during an assisted transfer from bed-to-wheelchair, the patient and nursing staff reported hearing a 'popping sound' suspected in the thoracic region of the back. The patient immediately experienced a flushing of his upper body along with diaphoresis, piloerection, hypotension $(\mathrm{BP}=87 / 53)$ and tachycardia (Pulse of 115). The episode resolved within minutes after the patient was returned to his bed and was placed in a recumbent position. A complete physical examination

Correspondence: JP Beard, MD did not reveal any changes from those present on admission. The patient's indwelling Foley catheter was removed and was replaced. Thoracic and lumbosacral spine radiographs were obtained and revealed severe osteopenia but no evidence of fracture or subluxation. The urine culture grew Serratia marsecens present in $<100 \mathrm{~K}$ colonies and the patient was started prophylactically on an oral antibiotic.

On subsequent days whilst lying supine in bed, the patient experienced no further such episodes but he did complain of shortness of breath and palpitations. $\mathrm{He}$ had a cardiac dysrhythmia, and a 12-lead electrocardiogram confirmed the presence of a bigeminy-trigeminy rhythm with unifocal premature ventricular contractions (PVCs), incomplete right bundle branch block and a previously noted right axis deviation. An echocardiogram was normal except for dysrhythmia. Chest radiographs revealed mild pulmonary edema. Cardiology consultants determined that this rate-controlled asymptomatic rhythm did not require medical intervention. The pulmonary edema resolved spontaneously in subsequent days.

The patient proceeded to experience autonomic dysreflexia (AD) with hypertension (occasional hypotensive episodes), tachy- or bradycardia, and cephalgia along with the previously noted symptoms when placed in a seated position. His symptoms resolved upon reclining to the supine position.

Further examination revealed a mildly enlarged right testis and ultrasonography demonstrated epididymal cysts and a small hydrocele. Follow-up urine cultures grew Serratia and the patient was treated for 21 days with appropriate intravenous antibiotics. Despite antibiotic treatment and subsequent negative urine cultures, the patient continued to experience episodes of positionally-induced AD. 
During the course of evaluation and treatment of the patient's $\mathrm{AD}$, multiple etiologies and treatments were pursued, including: bladder and bowel decompression, inspection of the skin for breakdown or irritation, assessment of the position of the scrotum while sitting, examination for intra-abdominal pathology (including KUB and ultrasonography for gastrointestinal, kidney or gallbladder disease) and laboratory evaluation (CBC and serum chemistries). These evaluations revealed no evidence of pathology. Of note on physical examination was the absence of AD symptoms on passive flexion of the hips while the patient was supine in bed but present when the patient was seated. Laboratory studies revealed an elevated alkaline phosphatase level and therefore pelvic and hip radiographs were obtained to evaluate the possible presence of heterotopic ossification (HO). These radiographs demonstrated an old fracture at the right anterior superior iliac spine, diffuse osteopenia, osteitis pubic symphysis, and an old bone infarct of the right femur. There was no evidence of $\mathrm{HO}$ or of a sacral fracture (Figure 1)

A Technetium $99 \mathrm{~m}$ bone scan was obtained which revealed increased uptake in the cervical spine (the site of the original SCI trauma), the sacroiliac joints and

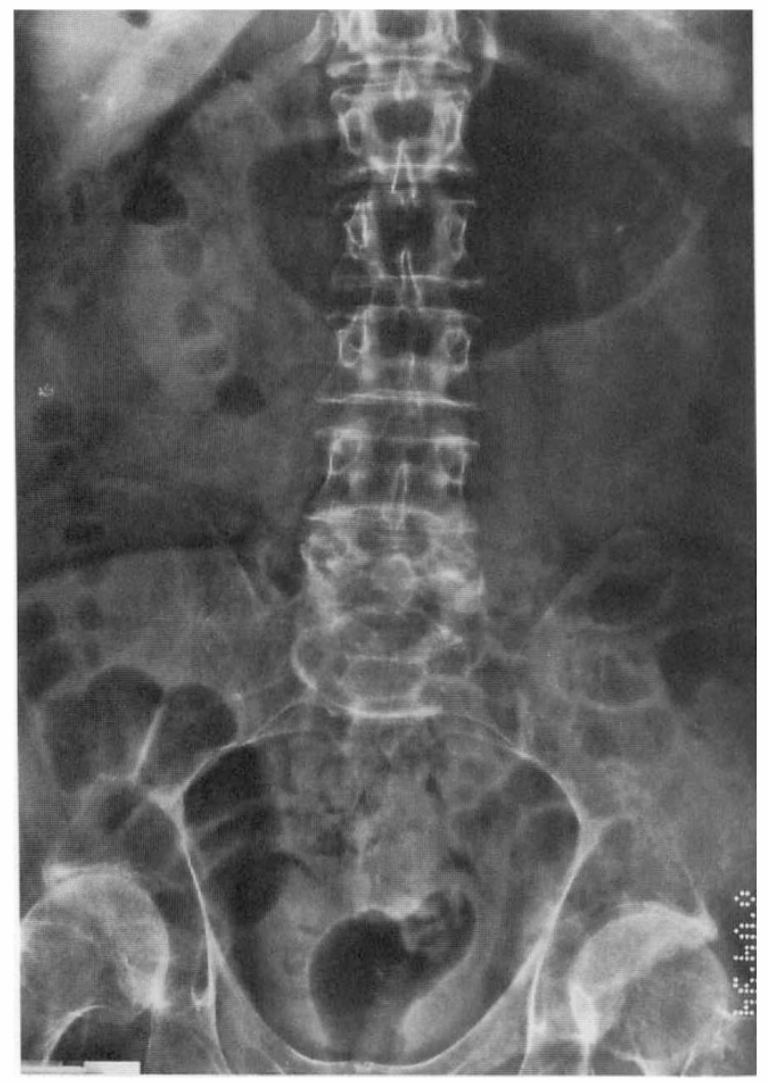

Figure 1 Roentgenogram of pelvis demonstrates osteopenia without evidence of sacral fracture the sacrum. The sacral pattern of uptake was in an $\mathrm{H}$ pattern most consistent with a stress fracture of the sacrum $^{1}$ (Figure 2). An Indium WBC-tagged study was performed to rule out osteomyelitis of the pelvis; there was no evidence of infection.

The patient was treated with a progressive sitting program consisting of a gradual increase in both the angle of incline and the time spent sitting up in his wheelchair. He was ultimately able to tolerate sitting for prolonged periods of time without symptoms of AD. He was discharged home with occasional mild episodes of $\mathrm{AD}$ which eventually resolved.

\section{Discussion}

Autonomic dysreflexia (AD) may be seen in approximately $85 \%$ of spinal cord injured patients with injuries above the major splanchnic outflow in response to noxious stimuli below the level of the lesion. $^{2-7}$ Observation, experimental models and postulates of the pathophysiologic mechanism(s) can be found in published literature. Most suggest the mechanism involves the loss of the descending central sympathetic inhibitory activity. Although numerous stimuli such as tight clothing, ${ }^{7}$ ingrown toenails, ${ }^{8}$

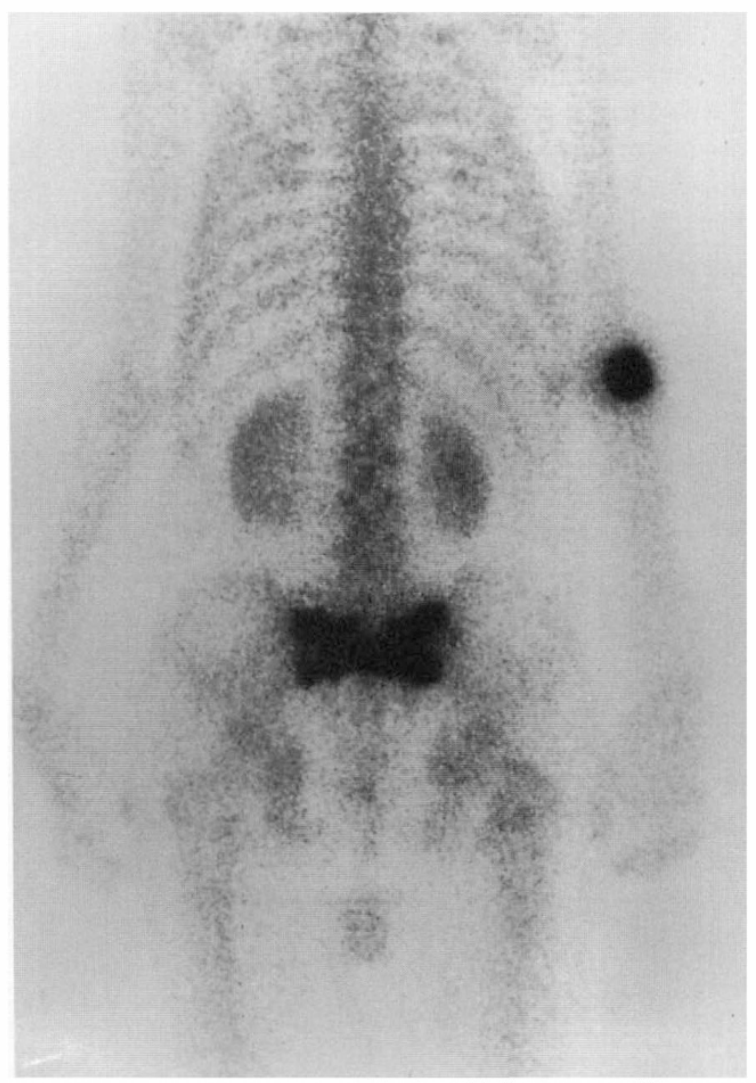

Figure 2 Delayed posterior image of sacral portion of whole body Technetium $99 \mathrm{~m}$ bone scan demonstrates increased bone uptake in H-pattern characteristic of sacral insufficiency stress fracture 
pulmonary emboli, ${ }^{9}$ or an acute surgical abdomen ${ }^{10,11}$ have been cited as initiating AD, clinical observations reveal bladder and bowel distention as the two most frequent stimuli causing $\mathrm{AD}$ in the clinical setting. ${ }^{2,7}$

Postural AD has been previously reported. Abouleish et $a l^{12}$ described AD stimulation by movement in a parturient, however, uterine contractions could have been the inciting stimuli in this case., ${ }^{2,13}$ Khurana reported three subjects with orthostatic hypotensioninduced $\mathrm{AD}$, alerting the clinician to the potentially counterproductive effect of sitting the patient upright and to the need for medical management of hypotension-induced AD. ${ }^{14}$ Although the symptoms were effectively treated in that series of patients, the stimulus for hypotension was presumed to be autonomic insufficiency; no evaluation of bony pathology was recorded. Thus, it is uncertain as to the underlying pathology of $\mathrm{AD}$ in these long-term tetraplegic patients who would undoubtedly have had osteopenia. Thompson and Witham reported a patient who noted AD symptoms with catheter changes, sudden changes of position, and following the administration of enemas. ${ }^{3}$

The potentially serious medical sequelae of $\mathrm{AD}$ as demonstrated in this case and others, cardiac dysrhythmia ${ }^{5}$ and pulmonary edema, ${ }^{15}$ compels the clinician to identify and irradicate the noxious stimulus(i). After thorough evaluation, we suspected two stimuli to be the most likely cause of this patient's $\mathrm{AD}$, a urinary tract infection and a possible epididymitis. The appropriate treatment of these infections along with the positional nature of the $\mathrm{AD}$ argued against either of these as being the etiology of his symptoms. The clinical history of a 'popping sound' in the vertebrae just prior to the first episode of $\mathrm{AD}$ raised the question of a fracture and/or subluxation of the vertebrae as the symptom's source. Negative radiographs of the thoracic and lumbosacral spine made this unlikely. With the elevated alkaline phosphatase level, heterotopic ossification (HO) was next suspected as the stimulating agent, yet this was not confirmed by the radiographs or the bone scan. The Technetium bone scan did reveal the characteristic H-pattern felt to be diagnostic of a stress fracture of the pelvis. ${ }^{1}$ Once the patient's sacral stress fracture was diagnosed, the appropriate treatment regimen could then be utilized to manage his AD.

\section{Conclusion}

The increased risk for fracture of the extremities as a result of osteopenia in individuals with spinal cord injury is well-documented. ${ }^{16}$ What may not be appreciated, however, is the degree to which this increased risk for fracture may also exist in other osseous structures. The patient presented in this case apparently sustained sufficient trauma in the recent past to cause a pelvic stress fracture even though there was no history of any such event(s). Plain radiographs failed to reveal evidence of fractures of the sacrum but a stress fracture was demonstrated by the bone scan. The necessity for continuing to search for the cause of an episode of AD in an SCI patient has been demonstrated in this case. It is only after the inciting stimulus has been identified that the rendering of appropriate medical treatment can be assured.

\section{Acknowledgements}

The authors wish to thank Laura Golightly for literary assistance.

\section{References}

1 Jones DN, Wycherley AG. Bone scan demonstration of progression of sacral insufficiency stress fracture. Australas Radiol 1994; 38 (2): 148 - 150

2 Bloch RF. Autonomic Dysfunction In: Bloch RF, Basbaum M (eds) Management of Spinal Cord Injuries. Williams \& Wilkins: Baltimore 1986, pp 149-163.

3 Thompson CE, Witham AC. Paroxysmal hypertension in spinalcord injuries. New Eng J Med 1948; 239 (8): 291 - 294.

4 Guttman L, Whitteridge D. Effects of bladder distention on autonomic mechanisms after spinal cord injuries. Brain 1947; 70: $361-404$.

5 Trop CS, Bennett CJ. The evaluation of autonomic dysreflexia. [Review]. Semin Urol 1992; 10 (2): 95-101.

6 Kurnick NB. Autonomic hyperreflexia and its control in patients with spinal cord lesions. Ann Intern Med 1966; 44: 678-686.

7 Colachis SC. Autonomic hyperreflexia with spinal cord injury. $J$ Am Paraplegic Society 1992; 15 (3): 171-186.

8 Lindan R, Joner B, Freehafer AA, Hazel C. Incidence and clinical features of autonomic dysreflexia in patients with spinal cord injury. Paraplegia 1980; 18: 285-292.

9 Colachis SC. Autonomic hyperreflexia in spinal cord injury associated with pulmonary embolism. Arch Phys Med Rehabil 1991; 72: 1014-1016.

10 Charney KJ, Juler GL, Comarr AE. General surgery problems in patients with spinal cord injuries. Arch Surg 1975; 110: $1083-$ 1088.

11 Neumayer LA, Bull DA, Mohr JD, Putnam CW. The acutely affected abdomen in paraplegic spinal cord injury patients. Ann Surg 1990; 212: 561 - 566.

12 Abouleish EI, Hanley ES, Palmer SM. Can epidural fentanyl control autonomic hyperreflexia in a quadriplegic parturient? Anesth Analg 1989; 68: 523 - 526.

13 Tabsh KMA, Brinkman CR III, Reff RA. Autonomic dysreflexia in pregnancy. Obstet Gynecol 1982; 60: 119-121.

14 Khurana RK. Orthostatic hypotension-induced autonomic dysreflexia. Neurology 1987; 37: $1221-1224$.

15 Kiker JD, Woodside Jr, Jelinek CE. Neurogenic pulmonary edema associated with autonomic dysreflexia. J Urol 1982; 128: $1038-1039$.

16 Keating JF, Kerr M, Delargy M. Minimal trauma causing fractures in patients with spinal cord injury. Disabil Rehabil 1992; 14 (2): $108-109$. 